\title{
BMJ Open Long-term weight loss success and the health behaviours of adults in the USA: findings from a nationally representative cross-sectional study
}

\author{
Kerem Shuval (D) , ,2,3 Elisa Morales Marroquin, ${ }^{4,5}$ Qing Li, ${ }^{6}$ Gregory Knell, ${ }^{4,5,7}$ \\ Kelley Pettee Gabriel, ${ }^{8}$ Jeffrey Drope,${ }^{9}$ Amy L Yaroch, ${ }^{10}$ Karen G Chartier, ${ }^{11}$ \\ Bob M Fennis, ${ }^{12}$ Mahmoud Qadan $^{1}$
}

To cite: Shuval $\mathrm{K}$,

Marroquin EM, Li Q, et al. Longterm weight loss success and the health behaviours of adults in the USA: findings from a nationally representative crosssectional study. BMJ Open 2021;11:e047743. doi:10.1136/ bmjopen-2020-047743

- Prepublication history for this paper is available online. To view these files, please visit the journal online (http://dx.doi org/10.1136/bmjopen-2020047743).

Received 07 December 2020 Accepted 22 June 2021
Check for updates

(C) Author(s) (or their employer(s)) 2021. Re-use permitted under CC BY-NC. No commercial re-use. See rights and permissions. Published by BMJ.

For numbered affiliations see end of article.

Correspondence to Professor Kerem Shuval; kerem.shuval@gmail.com

\section{ABSTRACT}

Objective To describe the relationship between longterm weight loss (LTWL) success and lifestyle behaviours among US adults.

Design Serial cross-sectional data from National Health and Nutrition Examination Survey cycles 2007-2014.

Setting and participants Population-based nationally representative sample. The analytic sample included 3040 adults aged 20-64 years who tried to lose weight in the past year.

Measures Participants were grouped into five LTWL categories $(<5 \%, 5 \%-9.9 \%, 10 \%-14.9 \%, 15 \%-19.9 \%$ and $\geq 20 \%$ ). Lifestyle-related behaviours included the following: alcohol intake, physical activity, smoking, fastfood consumption, dietary quality (Healthy Eating Index (HEI)) and caloric intake. Multivariable regression was employed adjusting for age, sex, race/ethnicity, marital status, education, household income and size, current body mass index and self-reported health status. Results Individuals in the 15\%-19.9\% LTWL group differed significantly from the reference group $(<5 \% \mathrm{LTWL})$ in their physical activity and dietary quality (HEI) but not caloric intake. Specifically, they had a higher HEl score $(\beta=3.19 ; 95 \% \mathrm{Cl} 0.39$ to 5.99$)$ and were more likely to meet physical activity guidelines $(\mathrm{R}=1.99 ; 95 \% \mathrm{Cl}$ 1.11 to 3.55$)$. In comparison, the $\geq 20 \%$ LTWL group was significantly more likely to smoke $(\mathrm{OR}=1.63 ; 95 \% \mathrm{Cl} 1.03$ to 2.57 ) and to consume lower daily calories ( $\beta=-202.91$. $95 \% \mathrm{Cl}-345.57$ to -60.25$)$ than the reference group; however, dietary quality and physical activity did not significantly differ.

Conclusion Among a national sample of adults, a higher level of LTWL success does not necessarily equate to healthy weight loss behaviours. Future research should attempt to design interventions aimed at facilitating weight loss success while encouraging healthy lifestyle behaviours.

\section{INTRODUCTION}

Over the past two decades, the prevalence of obesity among US adults increased from $30.5 \%$ in $1999-2000$ to $42.4 \%$ in $2017-2018$. $^{1}$ Whereas human physiology and genes have remained constant, the environment has

\section{Strengths and limitations of this study}

- This study includes four cycles of nationally representative data among US adults attempting to lose weight.

- Few studies have examined (un)healthy weight loss practices among adults with varying levels of longterm weight loss success.

- The study design is cross-sectional which prohibits determining a temporal relationship and reaching causal inferences.

- Despite using validated instruments, many study variables are based on participant report which could lead to overreporting (eg, physical activity) or underreporting (eg, alcohol consumption).

drastically changed to one that is conducive to overeating and physical inactivity. ${ }^{2-4}$ Specifically, the introduction of energysaving technology (eg, motorised transport) alongside constant environmental cues encouraging increased caloric intake of energy-dense nutrient-poor foods and beverages has led to positive energy balance. ${ }^{25-7}$ That is, energy intake exceeds expenditure, which leads to weight gain and often an obesity weight status. ${ }^{7-9}$ Obesity is related to increased risks for type 2 diabetes, cardiovascular disease, some cancers and premature mortality. ${ }^{1}{ }^{10} 11$ Beyond adverse health outcomes, obesity impacts the medical care system with direct costs related to medical treatment as well as indirect costs, such as productivity loss. ${ }^{1}$

Once weight gain has occurred, it is extremely difficult to lose weight with only $20 \%$ of the population able to successfully achieve long-term weight loss (LTWL); defined as losing $5 \%-10 \%$ of initial body weight within 6 months and maintaining it for a year or longer. ${ }^{12}$ Those successful 
have achieved LTWL by significantly changing their diet (eg, improved diet quality and smaller portion sizes) via self-monitoring of weight, and by habitually engaging in health-promoting physical activity. ${ }^{13}{ }^{14}$ In addition to eating a healthful diet (eg, increased fruits and vegetables) and being physically active, other behaviours have been found to positively or negatively affect LTWL. For example, cigarette smoking has been shown to decrease appetite, thereby positively contributing to LTWL, ${ }^{15} 16$ while simultaneously increasing cancer and premature mortality risk. ${ }^{1718}$ Thus, engaging in unhealthy behaviours such as smoking may lead to weight loss while having adverse health consequences. ${ }^{19}$ However, it is unclear whether adults who reach higher levels of LTWL are in fact engaging in healthy weight loss practices to achieve success. Studies on this topic have primarily focused on adolescents, ${ }^{20}$ with scant research centred on adults, particularly using nationally representative samples.

Therefore, the main goal of this study is to examine the relationship between LTWL and associated health behaviours among adults concerned with their weight. Specifically, we seek to identify lifestyle behaviours related to LTWL success, including physical activity, smoking, alcohol consumption, fast-food consumption, overall dietary quality and caloric intake. To this end, we use data from the National Health and Nutrition Examination Survey (NHANES), which has extensive information pertaining to health behaviours from a nationally representative sample of US adults.

\section{METHODS}

\section{Data and participants}

Data for the study were derived from four cycles (2007-2008, 2009-2010, 2011-2012 and 2013-2014) of NHANES, which is operated by the National Center for Health Statistics (NCHS). It consists of biannual multistage cross-sectional data collection on the health and nutritional status of adults and children in the USA. ${ }^{21}$ All study participants provide informed consent. In this study, we focus on adult participants who were overweight or obese at one point during their life, and who aimed to lose weight.

To this end, a total of 4380 participants meeting the following criteria were considered for inclusion: aged 20-64 years ${ }^{12}$; who tried to lose weight in the past year; who were not underweight (body mass index (BMI) $<18.5 \mathrm{~kg} / \mathrm{m}^{2}$ ), had a maximum BMI $\geq 25 \mathrm{~kg} / \mathrm{m}^{2}$, not pregnant and free of confusion and memory problems or physical/mental/emotional limitations. Adhering to a complete case analytic approach, ${ }^{12}$ a total of 1340 observations were omitted due to missing information on the independent or dependent variables as well as covariates. This resulted in 3040 participants included in the analytic sample. Outliers were not removed from the dataset to minimise type 1 error. $^{22}$

\section{Dependent and independent variables}

The primary independent variable was LTWL success. Consistent with previous research on this topic, ${ }^{5} 1223$ LTWL was calculated by subtracting participants' reported current weight or weight 1 year ago (the highest of the two) from their maximum weight. This was then divided by the maximum weight and multiplied by 100 to reach weight loss percentages. LTWL percentages were then grouped into the following clinically meaningful categories: $<5 \%$ (reference group), $5 \%-9.9 \%, 10 \%-14.9 \%$, $15 \%-19.9 \%$ and $\geq 20 \% .{ }^{1223} 24$

The dependent variables pertained to lifestyle behaviours. Specifically, current smoking status was based on the blood cotinine levels using an established cut-off of $3.08 \mathrm{ng} / \mathrm{mL} .^{25}$ Alcohol intake was based on the reported average number of drinks per day consumed in the past 12 months. Participants were defined as meeting alcohol guidelines for moderate use if consuming one alcoholic drink or less per day for women or two drinks or less daily for men. ${ }^{26}$ Leisure-time physical activity was based on the reported frequency (times) per week and duration (min) per day within moderate and vigorous intensity categories. ${ }^{27}$ For each intensity category, the product of the reported duration and frequency was multiplied by 4 and 8 metabolic equivalent of tasks (METs) for moderate and vigorous intensity, respectively, and expressed as MET $\mathrm{min} /$ week. The intermediate scores were summed to derive a final summary estimate of leisure-time physical activity. A threshold of $\geq 500$ MET min/week was applied to categorise participants as meeting (or not) the 2018 Physical Activity Guidelines ${ }^{28}$ (3.3 METs $\times 150 \mathrm{~min} /$ week; where 3.3 METs are equivalent to walking for pleasure at approximately 3.0 miles per hour). ${ }^{29}$

Moreover, the number of meals consumed at a fast-food or pizza place in the last 7 days was based on self-report. ${ }^{30}$ For multivariable analysis, responses were grouped into the following categories based on consumption per week: $0-1,2-3,4-6,7-10$ and $>10 .{ }^{31} 32$ In addition, participants' dietary quality was determined via the Healthy Eating Index (HEI)-2015, which assesses the degree of alignment with the 2015-2020 Dietary Guidelines for Americans. ${ }^{33} 34$ The HEI score ranges from 0 to 100 with the maximum score indicating complete adherence to dietary recommendations. The HEI-2015 was developed by the US Department of Agriculture Center for Nutrition Policy and Promotion; and then the National Cancer Institute's methodology was employed to compute the HEI scores from the first day of 24-hour dietary recall data. ${ }^{35}{ }^{36}$ Alongside dietary quality, total daily calories (kcal) intake was regarded as a separate dependent variable.

\section{Covariates}

The following covariates were taken into account in multivariable analyses based on the literature ${ }^{512}$ : age (years), sex (women/men), race/ethnicity (non-Hispanic white, non-Hispanic black, Hispanics and others), marital status (married/living together, widowed, divorced/separated and never married), college education (no/yes), 
household size (continuous), annual household income (<US\$20 000, US\$20 000-US\$44 999, US\$45 000-US\$74 999 and $\geq$ US $\$ 75000$ ), ${ }^{14}$ current BMI (continuous) and self-reported health status (poor, fair, good, very good and excellent).

\section{Statistical analysis}

Weighted descriptive statistics were employed to depict participants' baseline characteristics. The weighted mean (SE) of continuous dependent variables (eg, HEI-2015) and the prevalence of categorical-dependent variables (eg, smoking) was summarised by LTWL groups, and a $\mathrm{p}$ value was computed to test for statistical significance across categories using the adjusted Wald test. In addition, multivariable logistic regression was used when the dependent variables were dichotomous (eg, meeting physical activity guidelines (no/yes)), whereas ordinary least square (OLS) regression models were computed when the dependent variables were continuous (eg, HEI). Furthermore, when the dependent variable was the ordinal (ie, number of fast-food meals consumed per week: $0-1,2-3$, $4-6,7-10$ and $>10$ ), ordered logistic regression was used, which should be interpreted as transitioning to a higher category in comparison to remaining in the same category. ${ }^{37}$ Separate multivariable models were computed for each dependent variable while adjusting for covariates: age, sex, race/ethnicity, marital status, college education, annual house income and household size, current BMI and self-reported health status. In logistic regression models, the point estimates were ORs, whereas in OLS they were beta coefficients $(\beta)$. The measure of precision was $95 \%$ CIs. STATA 1SE V.15.1 (Stata-Corp LP) was used for analysis and appropriate survey weights were used to account for the complex sampling design of NHANES. ${ }^{38}$

\section{Patients and public involvement}

Neither the patients nor the public were involved in the study design, data collection, analytics or write up.

\section{RESULTS}

Participant characteristics are presented in table 1. Briefly, more than half $(56.7 \%)$ were women, $70.1 \%$ were nonHispanic white, 13.3 were Hispanics and $10.8 \%$ were nonHispanic blacks. In addition, $21.0 \%$ were current smokers and $51.9 \%$ met moderate alcohol drinking guidelines. Almost two-thirds $(65.2 \%)$ met physical activity guidelines. The average daily caloric intake was $2175.4 \mathrm{kcal}$, and the mean overall dietary quality, as measured by the HEI-2015, was 51.0/100. Participants' current mean BMI $\left(\mathrm{kg} / \mathrm{m}^{2}\right)$ was 31.8 and they lost 5.9 pounds, on average, from the previous year. Moreover, a total of $39.9 \%$ achieved LTWL success of $\geq 5 \%$. Furthermore, descriptive statistics of the dependent variables (health behaviours, dietary quality and caloric intake) by LTWL categories are depicted in table 2. Daily caloric intake, diet quality and physical activity differed significantly by LTWL $(p<0.05$ for all; see table 2). For example, the dietary
Table 1 Weighted characteristics of study sample: NHANES 2007-2014 $(n=3040)$

\begin{tabular}{ll}
\hline Characteristics & $\%^{*}$ \\
\hline Women & $56.7 \%$ \\
\hline Age: mean (SE) & $42.4(0.3)$ \\
Weight change-1 year (lbs)†: mean (SE) & $-5.9(0.4)$ \\
\hline Current BMI $\left(\mathrm{kg} / \mathrm{m}^{2}\right)$ : mean (SE) & $31.8(0.2)$ \\
\hline LTWL & \\
\hline $0 \%-4.9 \%$ & $60.1 \%$ \\
\hline $5 \%-9.9 \%$ & $20.8 \%$ \\
\hline $10 \%-14.9 \%$ & $9.7 \%$ \\
\hline $15 \%-19.9 \%$ & $4.4 \%$ \\
\hline$\geq 20 \%$ & $5.0 \%$ \\
\hline Race/ethnicity & \\
\hline Non-Hispanic white & $70.1 \%$ \\
\hline Hispanic & $13.3 \%$ \\
\hline Non-Hispanic black & $10.8 \%$ \\
\hline Other & $5.7 \%$ \\
\hline
\end{tabular}

Marital status

\begin{tabular}{ll} 
Married/living together & $67.1 \%$ \\
Widowed & $1.0 \%$ \\
\hline Divorced/separated & $14.0 \%$ \\
Never married & $17.9 \%$ \\
College educated & $36.3 \%$
\end{tabular}

Self-reported health status

\begin{tabular}{ll} 
Excellent & $9.2 \%$ \\
Very good & $33.0 \%$ \\
Good & $42.7 \%$ \\
Fair & $13.1 \%$ \\
Poor & $1.9 \%$ \\
Household size: mean (SE) & $3.1(0.0)$ \\
Annual household income & \\
<US\$20000 & $8.9 \%$ \\
US\$20 000-US\$44999 & $20.1 \%$ \\
US\$45 000-US\$74999 & $25.7 \%$ \\
\hline US $\$ 75000$ & $45.2 \%$
\end{tabular}

Fast-food meals-past week

\begin{tabular}{ll}
\hline $0-1$ & $53.8 \%$ \\
\hline $2-3$ & $27.5 \%$ \\
\hline $4-6$ & $11.7 \%$ \\
\hline $7-10$ & $5.2 \%$ \\
\hline 10 & $1.8 \%$ \\
\hline HEl-2015§: mean (SE) & $51.0(0.4)$ \\
\hline Kcal: mean (SE) & $2175.4(18.1)$ \\
\hline Smokingף & $21.0 \%$ \\
\hline Moderate alcohol** & $51.9 \%$ \\
\hline Physical activity guidelines†† & $65.2 \%$ \\
\hline
\end{tabular}

Continued 
Table 1 Continued

\section{Characteristics}

$\%$ *

*If percentages do not reach $100 \%$ that is due to rounding. †Weight change from previous year to the current year. fLTWL was calculated by subtracting the reported weight 1 year ago or current weight (the higher of the 2), by the reported maximum weight, and then multiplying by 100 to receive a percentage.

§HEI-2015: indicates adherence to Dietary Guidelines for Americans. The score ranges from 0 to 100 with the highest score indicative of complete compliance with the guidelines. Kcal- total daily calories.

ПSmoking: current smoking status was based on blood cotinine levels using an established cut-off of $3.08 \mathrm{ng} / \mathrm{mL}$. ${ }^{* *}$ Moderate alcohol: participants were defined as meeting moderate drinking guidelines, if consuming one alcoholic drink or less per day for women or two drinks or less daily for men.

††MET minutes per week for leisure time physical activity. Meeting physical activity guidelines refers to $\geq 500 \mathrm{MET}$ $\mathrm{min} /$ week.

BMI, body mass index; HEI, Healthy Eating Index; LTWL, long-term weight loss; MET, metabolic equivalent of task.

quality (HEI-2015) of those achieving 15\%-19.9\% LTWL was higher than individuals in the $\geq 20 \%$ LTWL group (54.8/100 and 50.4/100, respectively).

The multivariable relations of LTWL success with the dependent variables are shown in table 3. In comparison to the reference group (LTWL $<5 \%$ ), the health behaviours of those achieving 5\%-9.9\% LTWL did not differ significantly except for fast-food intake (OR=0.80; 95\% CI 0.64 to 1.00$)$. Similarly, the behaviours of those achieving 10\%-14.9\% LTWL did not differ markedly from the reference. In contrast, participants in the $15 \%-19.9 \%$ LTWL group significantly differed in their physical activity and dietary quality, yet not caloric intake. Specifically, the 15\%-19.9\% LTWL group were almost twice $(\mathrm{OR}=1.99 ; 95 \% \mathrm{CI} 1.11$ to 3.55$)$ as likely to meet physical activity guidelines, and to have a higher HEI score $(\beta=3.19 ; 95 \%$ CI 0.39 to 5.99$)$. Finally, individuals in the $\geq 20 \%$ LTWL group were 1.63 times $(95 \%$ CI 1.03 to 2.57 ) more likely to be current smokers and to consume 202.91 fewer daily calories $(95 \% \mathrm{CI}-345.57$ to $-60.25)$ than the reference group. No significant differences were observed in dietary quality and other health behaviours (table 3).

\section{DISCUSSION}

This study significantly contributes to the literature by exploring the health behaviours of adults attempting to lose weight from a national sample of Americans using four waves of NHANES data. Few studies to date have examined whether various levels of weight loss success are achieved through healthy weight loss practices in adults. These findings show that higher levels of LTWL do not necessary equate to healthier behaviours. More specifically, our analysis reveals that adults in the 15\%-19.9\% LTWL category likely achieved this success through healthy behaviours, whereas those losing more weight (LTWL of $\geq 20 \%$ ) appear to have attained success through some behaviours that were likely detrimental to their health. More specifically, the 15\%-19.9\% LTWL group had higher odds to eat a higher quality diet and to meet physical activity guidelines. Notably, participants who achieved LTWL of $\geq 20 \%$ smoked more and consumed

Table 2 LTWL success and lifestyle behaviours of adults attempting to lose weight : bivariate analysis $†$

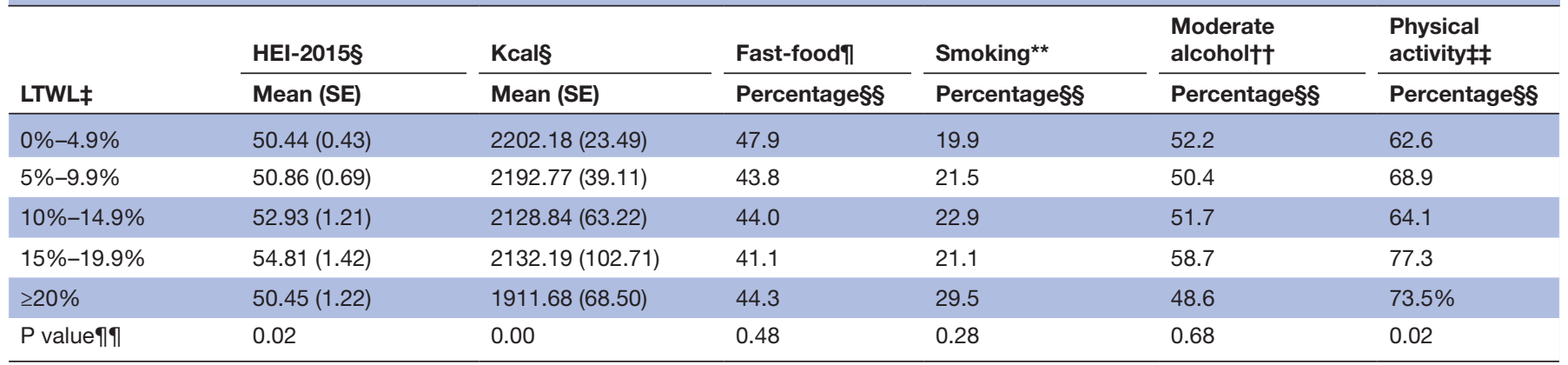

${ }^{*}$ NHANES data 2007-2014 ( $\left.\mathrm{n}=3040\right)$.

+Weighted descriptive statistics were employed to describe the mean (SE) for continuous variables and percentage for categorical variables by LTWL categories. fLTWL was calculated by subtracting the reported weight 1 year ago or current weight (the higher of the two), by the reported maximum weight, and then multiplying by 100 to receive a percentage.

\$HEl-2015: indicates adherence to Dietary Guidelines for Americans. The score ranges from 0 to 100 with the highest score indicative of complete compliance with the guidelines. Kcal- total daily calories.

१Number of fast-food or pizza place was dichotomised into $\geq 2$ (yes/no) meals in the past 7 days in bivariate analysis to ease interpretation

**Smoking: current smoking status was based on blood cotinine levels using an established cut-off of $3.08 \mathrm{ng} / \mathrm{mL}$.

††Alcohol: participants were defined as meeting moderate drinking guidelines, if consuming one alcoholic drink or less per day for women or two drinks or less daily for men.

$\ddagger$ MET minutes per week for leisure time physical activity. Meeting physical activity guidelines refers to $\geq 500 \mathrm{MET}$ min/week.

$\S \S$ Row percentage, that is, the percent of each categorical dependent variable (eg, smoking) in each LTWL group. For example, $19.9 \%$ of participants in the $0 \%-$ $4.9 \%$ LTWL group were current smokers.

Iा|Adjusted $\mathrm{p}$ value computed using the Wald test

HEI, Healthy Eating Index; LTWL, long-term weight loss; MET, metabolic equivalent of task; NHANES, National Health and Nutrition Examination Survey. 
Table 3 LTWL success and lifestyle behaviours of adults attempting to lose weight†: multivariable regressionł

\begin{tabular}{|c|c|c|c|c|c|c|}
\hline LTWL§ & HEI-2015ף & Kcalๆ & Fast-food $^{\star *}$ & Smokingtt & Moderate alcoholł¥ & Physical activity§§ \\
\hline $\begin{array}{l}\text { (Ref. } \\
\text { 0\%-4.9\%) }\end{array}$ & $\beta(95 \% \mathrm{Cl})$ & $\beta(95 \% \mathrm{Cl})$ & OR $(95 \% \mathrm{Cl})$ & OR $(95 \% \mathrm{Cl})$ & OR $(95 \% \mathrm{Cl})$ & OR $(95 \% \mathrm{Cl})$ \\
\hline $5 \%-9.9 \%$ & $0.25(-1.12$ to 1.61$)$ & $-15.54(-109.38$ to 78.30$)$ & $0.80^{*}(0.64$ to 1.00$)$ & 1.03 (0.79 to 1.35$)$ & 0.94 (0.76 to 1.16$)$ & 1.25 (0.96 to 1.62$)$ \\
\hline $10 \%-14.9 \%$ & $1.59(-0.92$ to 4.10$)$ & $-17.22(-140.93$ to 106.49$)$ & 0.94 (0.66 to 1.33$)$ & 1.35 (0.92 to 1.96$)$ & 1.01 (0.71 to 1.25$)$ & 1.04 (0.70 to 1.53$)$ \\
\hline $15 \%-19.9 \%$ & $3.19^{\star \star}(0.39$ to 5.99$)$ & $-47.66(-229.90$ to 134.59$)$ & 0.78 (0.50 to 1.22$)$ & 1.33 (0.81 to 2.19$)$ & 1.30 (0.85 to 1.99$)$ & $1.99^{\star}(1.11$ to 3.55$)$ \\
\hline$\geq 20 \%$ & $-0.27(-2.74$ to 2.19 & $-202.91^{* *}(-345.57$ to -60.25$)$ & $0.92(0.62$ to 1.36$)$ & $1.63^{*}(1.03$ to 2.57$)$ & 0.92 (0.60 to 1.42$)$ & $1.70(0.95$ to 3.01$)$ \\
\hline
\end{tabular}

${ }^{*} \mathrm{p}<0.05 ;{ }^{* *} \mathrm{p}<0.01$.

†NHANES data 2007-2014 ( $n=3040)$.

$\ddagger$ Separate regression models were employed for each dependent variable. Logistic and ordinal regression models were used when the variable was dichotomous (eg, meeting physical activity guidelines: yes/no) and ordered (fast-food), respectively. Ordinary least square regression models were used for continuous variables (HEI, kcal). NHANES weights were employed. Models adjusted for age, sex, race/ethnicity, marital status, college education, household income, household size, current BMI and self-rated health status. $\S L T W L$ was calculated by subtracting the reported weight 1 year ago or current weight (the higher of the two), by the reported maximum weight, and then multiplying by 100 to receive a percentage.

१HEI-2015: indicates adherence to Dietary Guidelines for Americans. The score ranges from 0 to 100 with the highest score indicative of complete compliance with the guidelines. Kcal: total daily calories.

${ }^{*}$ Number of fast-food or pizza place meals in the past 7 days: $0-1,2-3,4-6,7-10$ and $>10$.

††Smoking: current smoking status was based on blood cotinine levels using an established cut-off of $3.08 \mathrm{ng} / \mathrm{mL}$.

$\ddagger \ddagger$ Alcohol: participants were defined as meeting moderate drinking guidelines, if consuming one alcoholic drink or less per day for women or two drinks or less daily for men

$\S \S M E T$ minutes per week for leisure time physical activity. Meeting physical activity guidelines refers to $\geq 500 \mathrm{MET}$ min/week.

HEI, Healthy Eating Index; LTWL, long-term weight loss; MET, metabolic equivalent of task; NHANES, National Health and Nutrition Examination Survey.

significantly fewer calories. Thus, while reaching a higher degree of weight loss might lower cardiometabolic risk, ${ }^{12}$ achieving this goal by engaging in harmful behaviours, such as smoking, could adversely impact health and longevity. ${ }^{18}{ }^{39}$ Indeed, it increases cancer risk and shortens lifespans. ${ }^{17} 3940$

Evidence has shown that engaging in multiple health behaviours simultaneously reduces mortality risk from all-causes, cardiovascular disease and cancer. For example, Khaw et $a t^{41}$ found that in comparison to those adhering to four healthy behaviours (physical activity, fruit and vegetable intake, not smoking and moderate drinking), not adhering to any healthy behaviours was associated with a fourfold increased risk for all-cause mortality. Unlike the study by Khaw et al, this study focuses on the health behaviours of individuals with various levels of LTWL success rather than mortality as an endpoint. ${ }^{41}$ A previous study by Wilson, ${ }^{42}$ examined the relationship between physical activity and diet to LTWL among NHANES participants, finding that those with $10 \%$ LTWL were more likely to engage in vigorous intensity physical activity and to have lower caloric intake. ${ }^{42}$ This study, however, differs from Wilson's study in the following two main ways: (1) this research uses four waves of NHANES versus two waves and (2) it focuses specifically on adult dieters who may experience specific weight control challenges, ${ }^{43}$ while employing a more nuanced approach by examining five levels of LTWL (consistent with previous research on this topic) ${ }^{12} 23$ versus a dichotomous LTWL variable. ${ }^{42}$

This exploration of multiple levels of LTWL revealed that those achieving the highest level of success might have attained it by engaging in detrimental practices (eg, smoking). Smoking has been found in the literature to suppress appetite and prevent overeating, subsequently leading to LTWL success, despite its harmful effects. ${ }^{39} 444^{5}$ Phelan et $a l^{45}$ in a population-based study, observed that higher odds of LTWL success were related to markedly more smoking. Indeed, in this study those reaching $\geq 20 \%$ LTWL were $63 \%$ more likely to be current smokers than those who were not successful in weight loss ( $<5 \%$ LTWL). Research among adolescents underscores that there is a relationship between unhealthy weight loss practices (eg, diet pill use, fasting) and substance use behaviours, such as tobacco and marijuana smoking. ${ }^{20}$ A longitudinal study by Haynos et $a t^{46}$ found that unhealthy weight control practices among adolescents persist into adulthood and therefore there is a need to design intervention programmes to decrease these practices in adolescence and young adulthood. This study was conducted among adults aged 42 years (on average), showing that those best at LTWL $(\geq 20 \%)$ likely achieved this not by being physically active and improving diet quality but via caloric reduction (202.9 fewer daily calories), which might have been the result (at least partly) of smoking's appetite suppression effects. This explanation, however, should be regarded as a supposition since variables needed to explain behavioural choices were not available in the dataset.

Although psychological variables that illuminate participants' decision-making are not available in the data, the psychological literature might shed light on these findings. Previous research has linked high levels of self-regulation to virtuous decision making in the health, education, and financial domains. ${ }^{54-49}$ Specifically, high self-regulation necessitating goal setting and planning to meet a priori objectives, overcoming impediments and the ability to delay immediate gratification, ${ }^{50} 51$ has been linked to reduced obesity and cardiovascular disease risk, as well as better employment prospects. ${ }^{52-55}$ In a previous study among NHANES participants of low-income, we found that participants in the highest LTWL category $(\geq 20 \%)$ 
were also $45 \%$ less likely to save money. ${ }^{5}$ Based on the previous research, we hypothesised that this group used significant cognitive efforts to achieve LTWL and therefore did not have enough 'mental resources' when it came to financial decision making. ${ }^{5}{ }^{43}$

The current results add to our prior findings by showing that highest LTWL category $(\geq 20 \%)$ might have achieved weight loss via lower caloric intake and more smoking which suppress appetite, thereby likely exerting less self-control when it came to making healthy food choices. Indeed, the dietary quality of participants in the high LTWL group resembled those of the reference group (ie, LTWL $<5 \%$ ). It is unclear from the current study, whether these decisions were implicit or explicit, ${ }^{56}$ thus future research using data with robust psychological variables to examine the above-mentioned suppositions is needed. In comparison, participants in the 15\%-19.9\% LTWL category appear to be more likely to engage in healthy behaviours to achieve their weight loss goal. It is plausible that these participants had a higher level of self-regulation than the $\geq 20 \%$ LTWL group, yet this assumption needs to be substantiated with direct measurement. Furthermore, based on the previous health psychology research, it is possible that the 15\%-19.9\% LTWL group might have had a mental framework of themselves (ie, self-schema) as being healthy weight maintainers, thereby facilitating healthy behaviours. ${ }^{57-59}$ The NHANES dataset, however, does not measure self-schemas, thus these explanations need to be empirically examined in future work.

Beyond lacking psychological variables, this study has additional limitations that should be noted. Specifically, many of the variables used are based on participant reported responses to questionnaires which often leads to either overreporting (eg, physical activity) or underreporting (eg, alcohol intake). However, validated instruments were employed using standard protocols, thus if misclassification has occurred it is likely non-differential which leads to results being biased towards the null. ${ }^{60}$ Hence, the observed point estimates might be weaker than the true difference. In addition, the study design is cross-sectional which impedes examining a temporal relationship between the exposure and outcome and subsequently inferring any causal relationships. Moreover, it should be noted that participants' weight (maximum, 1 year ago and now) used to calculate LTWL, was determined at one point in time. Although historical weight has been found to be relatively stable ${ }^{12}{ }^{61}$ a longitudinal approach is preferable and prevents recall bias. Additionally, while participants' self-reported health status was adjusted for in multivariable analysis, as with other similar NHANES studies, ${ }^{23} 42$ medical conditions (eg, diabetes and coronary heart disease) and subsequent prescribed medication, which could influence LTWL and health behaviours, were not accounted for in this study. Finally, since a complete case analytic approach was taken, ${ }^{12}$ the analytic sample did not include participants with missing information on the independent and dependent variables as well as covariates.

In summary, data from a nationally representative sample of US adults who tried to lose weight shows that higher LTWL does not necessary relate to healthy weight loss practices. Notably, those achieving the most LTWL $(\geq 20 \%)$ were more likely to smoke, and to have lower caloric intake without improved diet quality. In comparison, those achieving slightly less LTWL (15\%-19.9\%) were engaged in healthy behaviours, such as meeting physical activity guidelines and having a better-quality diet. Thus, higher LTWL does not necessarily reflect healthier lifestyle practices, which might in turn, adversely impact future health and welfare. Future research should focus on understanding mechanisms explaining healthy and unhealthy weight loss practices of adults. This will enable the design and implementation of interventions aimed at helping adults lose weight while improving future health outcomes.

\section{Author affiliations}

${ }^{1}$ School of Business Administration, Faculty of Social Sciences, University of Haifa, Haifa, Israel

${ }^{2}$ School of Public Health, Faculty of Social Welfare and Health Sciences, University of Haifa, Haifa, Israel

${ }^{3}$ The Cooper Institute, Dallas, Texas, USA

${ }^{4}$ Department of Epidemiology, Human Genetics, and Environmental Sciences, The University of Texas Health Science Center at Houston (UTHealth), Houston, Texas, USA

${ }^{5}$ The Center for Pediatric Population Health, The University of Texas Health Science Center at Houston (UTHealth), Dallas, Texas, USA

${ }^{6}$ American Cancer Society, Atlanta, Georgia, USA

${ }^{7}$ Children's Health Andrews Institute for Orthopaedics and Sports Medicine, Plano, Texas, USA

${ }^{8}$ Department of Epidemiology, School of Public Health, University of Alabama at Birmingham, Birmingham, Alabama, USA

${ }^{9}$ Department of Health Policy and Administration, University of Illinois at Chicago, Chicago, Illinois, USA

${ }^{10}$ Gretchen Swanson Center for Nutrition, Omaha, Nebraska, USA

${ }^{11}$ School of Social Work and Department of Psychiatry, Virginia Commonwealth University, Richmond, Virginia, USA

${ }^{12}$ Department of Marketing, Faculty of Economics and Business, University of Groningen, Groningen, The Netherlands

Contributors KS conceived and led all aspects of the study. EMM meaningfully contributed to the write-up. QL and KS contributed to methodology employed, analytic approach and/or analyses. EMM, QL, GK, KPG, JD, ALY, KC, BMF and MQ provided critical insight on the methodology and/or feedback pertaining to study design and participated in critical revisions of the manuscript.

Funding The authors have not declared a specific grant for this research from any funding agency in the public, commercial or not-for-profit sectors.

Competing interests None declared.

Patient and public involvement Patients and/or the public were not involved in the design, or conduct, or reporting, or dissemination plans of this research.

Patient consent for publication Not required.

Ethics approval This study received approval from the University of Haifa Institutional Review Board (IRB: 297/20), exempt status from the University of Texas Health Science Center's Committee for the Protection of Human Subjects (HSCSPH-17-0925) and exempt from the Morehouse School of Medicine's IRB.

Provenance and peer review Not commissioned; externally peer reviewed. 
Data availability statement Data are available in a public, open access repository. The current study used data from NHANES. It is publicly available and can be downloaded from the Centers for Disease Control and Prevention website: https:// wwwn.cdc.gov/nchs/nhanes/

Open access This is an open access article distributed in accordance with the Creative Commons Attribution Non Commercial (CC BY-NC 4.0) license, which permits others to distribute, remix, adapt, build upon this work non-commercially, and license their derivative works on different terms, provided the original work is properly cited, appropriate credit is given, any changes made indicated, and the use is non-commercial. See: http://creativecommons.org/licenses/by-nc/4.0/.

\section{ORCID iD}

Kerem Shuval http://orcid.org/0000-0001-9542-0910

\section{REFERENCES}

1 Centers for Disease Control and Prevention. Obesity is a common, serious and costly disease, 2020. Available: https://www.cdc.gov/ obesity/data/adult.html [Accessed 06 Jun 2020].

2 Katz DL. Perspective: obesity is not a disease. Nature 2014;508:S57.

3 Booth FW, Chakravarthy MV, Gordon SE, et al. Waging war on physical inactivity: using modern molecular ammunition against an ancient enemy. J Appl Physiol 2002;93:3-30.

4 Booth FW, Roberts CK, Laye MJ. Lack of exercise is a major cause of chronic diseases. Compr Physiol 2012;2:1143-211.

5 Shuval K, Fennis BM, Li Q, et al. Health \& Wealth: is weight loss success related to monetary savings in U.S. adults of low-income? Findings from a National Study. BMC Public Health 2019;19:1-7.

6 Shuval K, Leonard T, Drope J, et al. Physical activity counseling in primary care: insights from public health and behavioral economics. CA Cancer J Clin 2017;67:233-44.

7 González-Muniesa P, Mártinez-González M-A, Hu FB, et al. Obesity. Nat Rev Dis Primers 2017;3:17034.

8 Hill JO, Wyatt HR, Peters JC. Energy balance and obesity. Circulation 2012;126:126-32.

9 Hill JO, Wyatt HR, Peters JC, et al. The importance of energy balance. Eur Endocrinol 2010;9:111.

10 Tobias DK, Hu FB. The association between BMI and mortality: implications for obesity prevention. Lancet Diabetes Endocrino 2018;6:916-7.

11 Grundy SM. Obesity, metabolic syndrome, and cardiovascular disease. J Clin Endocrinol Metab 2004;89:2595-600.

12 Knell G, Li Q, Pettee Gabriel K, et al. Long-Term weight loss and metabolic health in adults concerned with maintaining or losing weight: findings from NHANES. Mayo Clin Proc 2018;93:1611-6.

13 Wing RR, Phelan S. Long-Term weight loss maintenance. Am J Clin Nutr 2005;82:222S-5.

14 Catenacci VA, Ogden LG, Stuht J, et al. Physical activity patterns in the National weight control registry. Obesity 2008:16:153-61.

15 Mineur YS, Abizaid A, Rao Y, et al. Nicotine decreases food intake through activation of POMC neurons. Science 2011;332:1330-2.

16 Audrain-McGovern J, Benowitz NL. Cigarette smoking, nicotine, and body weight. Clin Pharmacol Ther 2011;90:164-8.

17 Centers for Disease Control and Prevention. Smoking and cancer. smoking and cancer, 2020. Available: https://www.cdc.gov/tobacco/ campaign/tips/diseases/cancer.html [Accessed 27 Oct 2020].

18 Lariscy JT, Hummer RA, Rogers RG. Cigarette smoking and all-cause and cause-specific adult mortality in the United States. Demography 2018;55:1855-85.

19 Utter J, Denny S, Robinson E, et al. Identifying the 'red flags' for unhealthy weight control among adolescents: Findings from an item response theory analysis of a national survey. Int $J$ Behav Nutr Phys Act 2012;9:99.

20 Vidot DC, Messiah SE, Prado G, et al. Relationship between current substance use and unhealthy weight loss practices among adolescents. Matern Child Health J 2016;20:870-7.

21 Cetners for disease control and prevention. about the National health and nutrition examination survey. NHANES https://www.cdc.gov/ nchs/nhanes/about_nhanes.htm

22 Gress TW, Denvir J, Shapiro Jl. Effect of removing outliers on statistical inference: implications to interpretation of experimental data in medical research. Marshall J Med 2018;4.

23 Kraschnewski JL, Boan J, Esposito J, et al. Long-Term weight loss maintenance in the United States. Int J Obes 2010;34:1644-54.

24 Jensen MD, Ryan DH, Apovian CM. AHA/ACC/TOS guideline for the management of overweight and obesity in adults. Circulation 2013;2014:S102-38.
25 Benowitz NL, Bernert JT, Caraballo RS, et al. Optimal serum cotinine levels for distinguishing cigarette smokers and nonsmokers within different racial/ethnic groups in the United States between 1999 and 2004. Am J Epidemiol 2009;169:236-48.

26 National Institute on alcohol abuse and alcoholism (NIAAA). Drinking levels defined. drinking levels defined, 2020. Available: https:// www.niaaa.nih.gov/alcohol-health/overview-alcohol-consumption/ moderate-binge-drinking [Accessed 28 Oct 2020].

27 Du Y, Liu B, Sun Y. Trends in adherence to the physical activity guidelines for Americans for aerobic activity and time spent on sedentary behavior among US adults, 2007 to 2016. JAMA Netw Open 2019:2:e197597.

28 Department of health and human services. Physical activity guidelines Advisory Committee scientific report, 2018. Available: https://health.gov/paguidelines/second-edition/report/

29 Ainsworth BE, Haskell WL, Herrmann SD, et al. 2011 compendium of physical activities: a second update of codes and Met values. Med Sci Sports Exerc 2011;43:1575-81.

30 Centers for Disease Control and Prevention. DBQ_G. National health and nutrition examination survey, 2020. Available: https:// wwwn.cdc.gov/nchs/nhanes/2011-2012/dbq_g.htm [Accessed 11 Nov 2020].

31 Shuval K, Stoklosa M, Pachucki MC, et al. Economic preferences and fast food consumption in US adults: insights from behavioral economics. Prev Med 2016;93:204-10.

32 Anderson B, Rafferty AP, Lyon-Callo S, et al. Fast-Food consumption and obesity among Michigan adults. Prev Chronic Dis 2011;8:A71. accessed https://www.ncbi.nlm.nih.gov/pmc/articles/ PMC3136980/

33 Krebs-Smith SM, Pannucci TE, Subar AF, et al. Update of the healthy eating index: HEI-2015. J Acad Nutr Diet 2018;118:1591-602.

34 Institute NC. Developing the healthy eating index. healthy eating index, 2020. Available: https://epi.grants.cancer.gov/hei/developing. html [Accessed 23 Apr 2021].

35 Nguyen BT, Shuval K, Bertmann F, et al. The supplemental nutrition assistance program, food insecurity, dietary quality, and obesity among U.S. adults. Am J Public Health 2015;105:1453-9.

36 Guenther PM, Kirkpatrick SI, Reedy J, et al. The healthy eating Index-2010 is a valid and reliable measure of diet quality according to the 2010 dietary guidelines for Americans. J Nutr 2014;144:399-407.

37 Shuval K, Gabriel KP, Leonard T. Tv viewing and BMI by race/ ethnicity and socio-economic status. PLoS One 2013;8:e63579.

38 Shuval K, Li Q, Gabriel KP, et al. Income, physical activity, sedentary behavior, and the 'weekend warrior' among U.S. adults. Prev Med 2017; 103:91-7.

39 Banks E, Joshy G, Weber MF, et al. Tobacco smoking and allcause mortality in a large Australian cohort study: findings from a mature epidemic with current low smoking prevalence. BMC Med 2015;13:38.

40 Centers for Disease Control and Prevention. Tobacco-Related mortality. smoking and tobacco use, 2018. Available: http://www. cdc.gov/tobacco/data_statistics/fact_sheets/health_effects/tobacco_ related_mortality/ [Accessed 04 Oct 2018].

41 Khaw K-T, Wareham N, Bingham S, et al. Combined impact of health behaviours and mortality in men and women: the EPIC-Norfolk prospective population study. PLoS Med 2008;5:9.

42 Wilson P. Physical activity and dietary determinants of weight loss success in the US general population. Am J Public Health 2016;106:321-6.

43 Stroebe W, van Koningsbruggen GM, Papies EK, et al. Why most dieters fail but some succeed: a goal conflict model of eating behavior. Psychol Rev 2013;120:110-38.

44 White MA. Smoking for weight control and its associations with eating disorder symptomatology. Compr Psychiatry 2012;53:403-7.

45 Phelan S, Wing RR, Loria CM, et al. Prevalence and predictors of weight-loss maintenance in a biracial cohort: results from the coronary artery risk development in young adults study. Am J Prev Med 2010;39:546-54.

46 Haynos AF, Wall MM, Chen C, et al. Patterns of weight control behavior persisting beyond young adulthood: results from a 15-year longitudinal study. Int J Eat Disord 2018;51:1090-7.

47 de Ridder DTD, Lensvelt-Mulders G, Finkenauer C, et al. Taking stock of self-control: a meta-analysis of how trait self-control relates to a wide range of behaviors. Pers Soc Psychol Rev 2012;16:76-99.

48 Bradford D, Courtemanche C, Heutel G, et al. Time preferences and consumer behavior. J Risk Uncertain 2017;55:119-45.

49 Stoklosa M, Shuval K, Drope J, et al. The intergenerational transmission of obesity: the role of time preferences and self-control. Econ Hum Biol 2018;28:92-106.

50 Koch AK, Nafziger J. Self-Regulation through goal Setting*. Scand J Econ 2011:113:212-27. 
51 Mann T, de Ridder D, Fujita K. Self-Regulation of health behavior: social psychological approaches to goal setting and goal striving. Health Psychol 2013;32:487-98.

52 Van Lange PA, Kruglanski AW, Higgins ET. Handbook of theories of social psychology. Volume two. SAGE publications, 2011.

53 Sutter M, Kocher MG, Glätzle-Rützler D, et al. Impatience and Uncertainty: Experimental Decisions Predict Adolescents' Field Behavior. Am Econ Rev 2013;103:510-31.

54 Israel S, Caspi A, Belsky DW, et al. Credit scores, cardiovascular disease risk, and human capital. Proc Natl Acad Sci U S A 2014;111:17087-92.

55 de Oliveira ACM, Leonard TCM, Shuval K, et al. Economic preferences and obesity among a low-income African American community. J Econ Behav Organ 2016;131:196-208.
56 Elran-Barak R, Bar-Anan Y. Implicit and explicit anti-fat bias: the role of weight-related attitudes and beliefs. Soc Sci Med 2018;204:117-24.

57 Self-Schema: Oxford reference, 2020

58 Kendzierski D. A Self-Schema approach to healthy eating. J Am Psychiatr Nurses Assoc 2007;12:350-7.

59 Lee C-K, Liao L-L, Lai I-J, et al. Effects of a healthy-eater selfschema and nutrition literacy on healthy-eating behaviors among Taiwanese college students. Health Promot Int 2019;34:269-76.

60 Abramson JH, Abramson Z. Making sense of data: a self-instruction manual on the interpretation of epidemiological data. Oxford university press, 2001.

61 Casey VA, Dwyer JT, Berkey CS, et al. Long-Term memory of body weight and past weight satisfaction: a longitudinal follow-up study. Am J Clin Nutr 1991;53:1493-8. 